\title{
Politique
}

\section{Ouverture politique, transition démocratique et classe ouvrière en Argentine}

\section{Graciela Ducatenzeiler}

Numéro 12, automne 1987

Mouvements et acteurs

URI : https://id.erudit.org/iderudit/040568ar

DOI : https://doi.org/10.7202/040568ar

Aller au sommaire du numéro

Éditeur(s)

Société québécoise de science politique

ISSN

0711-608X (imprimé)

1918-6584 (numérique)

Découvrir la revue

Citer cet article

Ducatenzeiler, G. (1987). Ouverture politique, transition démocratique et classe ouvrière en Argentine. Politique, (12), 63-91. https://doi.org/10.7202/040568ar d'utilisation que vous pouvez consulter en ligne.

https://apropos.erudit.org/fr/usagers/politique-dutilisation/ 


\title{
OUVERTURE POLITIQUE, TRANSITION DÉMOCRATIQUE ET CLASSE OUVRIÈRE EN ARGENTINE
}

\author{
Graciela Ducatenzeiler \\ Université de Montréal
}

Au-delà des coïncidances chronologiques et au-delà des causes structurales communes, à la base de l'émergence et de la chute des régimes autoritaires, les processus de démocratisation en Amérique latine, surtout en ce qui concerne l'Argentine, le Brésil et l'Uruguay, se sont faits dans des contextes particuliers déterminés par l'histoire politique de chacun de ces pays. Ceci est vrai à un point tel que le concept même de démocratisation n'a pas un contenu univoque lorsqu'il est appliqué à chacune de ces réalités. En effet, l'utilisation du concept de démocratisation suppose que l'autoritarisme ait été un phénomène assez généralisé de leur vie politique. Cependant, même si cette affirmation est vraie pour l'Argentine et encore plus pour le Brésil, elle est fausse pour l'Uruguay qui depuis le début de ce siècle jusqu'à la fin des années soixante a connu une stabilité démocratique exceptionnelle en Amérique latine.

Sans remonter aux origines de ces histoires politiques nous pouvons affirmer qu'elles ont été marquées par le différent poids relatif de deux acteurs: l'État et la société civile. Au Brésil, l'État l'emporte sur la société civile en lui enlevant toute autonomie;

Politique, 12 (Automne 1987). 
en Argentine, la puissance de la société civile, divisée en forces de poids comparable, empêche la structuration de l'État en tant que terrain de compromis; en Uruguay l'équilibre entre société civile et État s'exprime par un fonctionnement politique semblable à celui de toute démocratie moderne. S'il fallait donc, identifier les acteurs politiques les plus importants dans chacun de ces trois pays, on aurait l'État au Brésil, les corporations en Argentine et les partis politiques en Uruguay.

Les formes de transitions politiques dans ces trois pays n'ont pas échappé à leur histoire. Au Brésil, les militaires ont contrôlé l'ouverture et ont imposé les conditions de la restauration des institutions; en Uruguay, malgré que l'initiative concernant l'ouverture soit venue des militaires, ceux-ci ont accepté le système de partis; la transition a été un pacte entre ceux qui contrôlaient l'État et la société civile à travers les partis politiques. En Argentine, par contre, la transition a adopté la forme d'une rupture précipitée par la guerre des Malouines où la transition s'est engagée malgré les convictions des militaires et malgré de fortes oppositions dans la société civile.

Comme l'affirme Gonzalez ${ }^{1}$ les facteurs internes qui ont déterminé les transitions configurent un triangle dont les sommets sont constitués par la pression populaire, les élites civiles et la direction des forces armées. Il s'agit de voir dans chaque situation concrète la structure des relations entre les sommets de ce triangle.

La majorité des analyses sur le Brésil signalent que le sommet dominant de la transition brésilienne est constitué par la direction militaire. Dans le cas uruguayen la plus grande partie de chercheurs ne voient pas de sommet dominant. En ce qui concerne l'Argentine

1. Luis González, Transición y restauración democrática, CIESU, Montevideo, 1985. 
le sommet principal est plutôt constitué par les militaires, mais non pas comme dans le cas brésilien à cause du contrôle qu'ils ont exercé sur le processus, mais à cause de leur échec qui a donné lieu à une situation de vide politique qui a permis la transition.

Cet article concerne la transition politique en Argentine et s'interroge notamment, sur le rôle de la classe ouvrière organisée dans le processus de transition. Nous nous intéressons, donc, à la structure des relations entre les pressions populaires, les élites civiles et la direction militaire, en mettant l'accent sur le premier élément.

Étant donné le consensus dans les milieux académiques concernant les déterminants internes de la transition, soit qu'elle n'a été ni une conquête populaire, ni une concession des militaires, ni le résultat d'un pacte entre ces derniers et les élites politiques, la littérature scientifique s'est surtout intéressée à l'étude du régime lui-même. L'espace politique ayant été réduit à la seule action du gouvernement, les chercheurs se sont surtout interrogés sur les causes de l'autoritarisme et les conséquences de ses politiques sur la structure de la société. L'étude des acteurs et de leurs transformations a commencé à se développer à partir du moment où ceux-ci ont récupéré la parole, c'est à dire, avec la restauration des institutions démocratiques. La centralité des syndicats dans la vie politique argentine depuis les années 50 jusqu'au coup d'État de 1976 fait de son étude une question d'intérêt non seulement académique mais aussi politique. L'affaiblissement de leur pouvoir pendant la période autoritaire, a-t-elle été une sorte d'interrègne ou plutôt l'expression d'un changement qualitatif dans leurs rapports avec d'autres acteurs sociaux? Les réponses à ces questions ont des conséquences importantes dans la construction d'un ordre démocratique en tant que déterminantes des politiques que les gouvernements civils adoptent et adopterons face aux syndicats. 
En Argentine, le renversement du gouvernement péroniste, en 1955, inaugura une nouvelle étape politique, étape dont les caractéristiques plus apparentes sont, sans conteste, l'instabilité et le recours répété au coup d'État. Cependant l'élément le plus original qui caractérisa la période qui s'ouvre en 1955 est peut être le rôle joué par le mouvement ouvrier organisé en tant qu'acteur central dans la scène politique. En effet, la nouvelle conjoncture transforma les syndicats en instruments uniques d'articulation des intérêts politiques de la classe ouvrière tout en gardant leur rôle traditionnel d'agents de négociation économique. Plusieurs facteurs sont à la base de cette centralité du mouvement ouvrier dans la scène politique: les nombreuses proscriptions subies par les partis politiques en général et le Parti péroniste, auquel les syndicats s'identifiaient, en particulier; l'instabilité des formules institutionnelles appliquées; le manque d'alternative aux échecs répétés et, fondamentalement, la faiblesse du système partisan argentin qui privilégie l'articulation corporatiste des intérêts de classe et de groupes au détriment de leur articulation à travers les partis politiques.

Cible ou promoteur, le mouvement ouvrier argentin participa à tous les enjeux, à tous les projets, à tous les conflits qui traversèrent la société argentine depuis 1955. Le régime autoritaire (19761983) le signala comme une des causes principales de la maladie qui affligeait le pays, "le pouvoir excessif des syndicats», et entreprenait des mesures à différents niveaux pour éliminer ce pouvoir. Dans cet article nous examinons ce qu'est devenu le syndicalisme argentin suite à sept ans de politiques destinées à l'affaiblir et quelle a été sa contribution au moment de l'ouverture du régime autoritaire.

Notre premier objectif est d'étudier l'influence du comportement de la classe ouvrière organisée dans l'ouverture politique qui s'insinue à partir de 1981 et qui se précipite comme conséquence 
de la guerre des Malouines de 1982. Ceci implique l'analyse des causes à la base de la libéralisation du régime autoritaire ainsi que du processus rapide d'ouverture politique qui s'est traduit par l'organisation subséquente des élections.

Le deuxième objectif est l'analyse des changements objectifs dans le mouvement ouvrier organisé à partir des nouvelles règles du jeu imposées par les militaires ainsi que les effets de ces changements sur le type d'action syndicale. Il s'agit d'évaluer l'influence des changements objectifs sur le pouvoir syndical.

Pour terminer nous nous intéresserons aux causes de la défaite politique du syndicalisme, via la défaite électorale du péronisme, dans les élections de 1983. Ceci nous conduit à l'articulation des transformations ou des constantes à l'intérieur du syndicalisme avec la transformation des anciennes forces politiques et l'émergence de nouveaux acteurs politiques dans l'ensemble de la société.

\section{Ouverture politique et classe ouvrière organisée}

L'ouverture politique n'a pas été arrachée au régime par une puissante opposition, mais elle a été plutôt le produit des conflits internes, de la perte de consensus à l'intérieur du propre régime concernant l'évaluation de leur gestion, la redéfinition des objectifs et notamment les moyens à utiliser pour les atteindre. La guerre des Malouines, qui aurait dû, dans le calcul de ses précurseurs, résoudre ou affaiblir ces conflits, a, au contraire, provoqué la destruction d'un régime qui tant bien que mal avait réussi à se tenir en place grâce, en partie, à la faiblesse de l'opposition. En effet, la désintégration interne du régime ne fut pas accompagnée du renforcement des oppositions; au moment du déclenchement de la guerre de l'Atlantique Sud, il n'en fallait pas beaucoup pour que le gouvernement perde le contrôle de l'ensemble de la société; le coup final provînt d'une action provoquée par les militaires 
mêmes: une guerre qui était perdue dès ses débuts et qui eut, par conséquent, l'effet d'un suicide politique.

La faiblesse de l'opposition ne peut pas cependant être interprétée comme une preuve de la légitimité dont jouissait le gouvernement. La légitimité issue du projet politique initial s'est effritée graduellement devant l'incapacité ou le désintéressement du régime à articuler les demandes provenant de l'extérieur y compris celles des secteurs qui avaient frappé à la porte des casernes pour s'associer ou pour collaborer avec les militaires dans le coup d'État. En effet, si l'intervention des militaires en 1976 fut reçue avec soulagement par de larges secteurs de la société et avec enthousiasme par une minorité, les diverses mesures prises par le gouvernement et, notamment leurs conséquences, ont vite fait déchanter et les uns et les autres.

Depuis 1981 le régime qui pendant quatre ans (1976-1980) avait offert l'image d'une extraordinaire unité, commençait à extérioriser des conflits produits par la naissance de fractions et de clivages personnels et idéologiques qui étaient des signes avantcoureurs de sa défaite. Le "Processus de réorganisation nationale», nom que les militaires avaient donné à leur gestion, s'appuyait fondamentalement sur son projet économique; son succès était donc la condition nécessaire à la transformation voulue des forces politiques et sociales et la justification de leur intervention en général et des moyens utilisés en particulier. L'échec du projet économique ne mettait donc pas simplement en question une partie de la gestion des militaires mais, plutôt, l'ensemble de leur gestion. C'est ainsi que le consensus interne concernant les principales idées et stratégies du gouvernement disparaissait et que les différents et conflits qui avaient été relégués pour permettre la construction de ce consensus, commencèrent à faire surface. Les forces armées se trouvaient divisées entre les «durs», partisans de la poursuite de la fermeture politique et du projet néo-libéral 
et les «modérés», plus ouverts au dialogue et à un certain nationalisme économique. Pris dans cette confrontation le régime se montra incapable d'arriver à un nouveau consensus quand à la voie à suivre.

C'est dans ce contexte de conflits irrésolus que Galtieri, avant-dernier président du «Processus», s'en va-t-en guerre, ses buts étant de retrouver l'unité interne des forces armées et de se donner une légitimité populaire. Mais la guerre perdue, les forces armées ne retrouvèrent ni leur unité ni la confiance populaire.

La défaite dans la guerre des Malouines ne fit qu'accélérer le processus de désintégration du régime tout en empêchant les militaires de poser des conditions à leur retrait du pouvoir. L'ouverture échappe au contrôle des forces armées qui doivent se remettre aux conditions d'une opposition pourtant fragile. Ironie de l'histoire, le régime autoritaire le plus dur qu'ait connu l'Argentine, fait place à un des processus électoraux le plus démocratiques à avoir lieu dans ce pays depuis la constitution de la République.

Cette ouverture et ensuite le processus de transition démocratique qui l'a suivi, eurent des caractéristiques très différentes par rapport à d'autres situations semblables qui ont marqué la période ouverte en 1955.

En effet l'autoritarisme précédent (1966-1973) avait dû faire face à partir de la moitié de sa gestion à des mobilisations très larges, constituées notamment par la jeunesse étudiante et les organisations ouvrières, dont le rôle a été déterminant pour la chute du régime. Cette fois-ci la classe ouvrière, pour la première fois, n'a pas été le protagoniste principal du changement de régime. Nous nous pencherons maintenant sur les causes qui ont déterminé la perte ou l'affaiblissement du puvoir syndical, ce qui est essentiel pour comprendre la victoire du Parti radical dans les élections de 1983. 


\section{Changements objectifs et pouvoir syndical}

Le coup d'État de 1976 met fin aux relations particulières qui avaient l'État et les syndicats en Argentine depuis le milieu des années 50. En effet un des objectifs principaux du gouvernement autoritaire était la transformation du système politique à travers la destruction du populisme. Il prétendait transformer l'ensemble de la société à partir d'une modification des conditions structurelles où le populisme puisait sa force. Il visait une transformation du système productif qui créerait de nouveaux acteurs économiques et politiques, de telle sorte que le rétablissement des institutions démocratiques ne reproduise pas la situation à laquelle il avait voulu remédier.

La destruction du populisme ouvrier impliquait une double intervention: a) une intervention politique qui s'attaquait directement à ce pouvoir, jugé excessif des syndicats; b) une intervention économique qui, indirectement mettrait le syndicalisme à sa place, à travers des changements importants dans le fonctionnement du marché du travail.

Le premier type d'intervention visait essentiellement la législation régissant les associations ouvrières et le contrôle de leur fonctionnement interne. Le deuxième type d'intervention impliquait une transformation révolutionnaire de la structure productive.

\section{L'intervention politique.}

Bien que les tentatives des gouvernements post-péronistes (à partir de 1955) de corroder la législation du travail populiste aient été infructueuses jusqu'en 1976, elles réussirent néanmoins, sans le chercher vraiment, à politiser davantage l'action ouvrière. Par conséquent, les syndicats se transformèrent de simples dépositaires des revendications économiques de la classe ouvrière en véritables groupes politiques de pression. Le régime populiste leur avait réservé un rôle fondamentalement économique, les régimes 
qui se voulaient post-populistes, leur donnaient des responsabilités politiques en les identifiant à un parti politique, le parti péroniste ou justicialiste, proscrit de la majorité des joutes électorales. C'est la coexistence de ces deux types de responsabilités qui donna toute sa force au syndicalisme argentin qui dès 1955 se servait des actions politiques pour appuyer sa négociation économique.

Les fréquentes proscriptions des partis politiques en général et, du Parti péroniste en particulier, ont constitué un atout pour l'action politique des syndicats; eux à la différence des premiers ont pu maintenir, tant bien que mal, selon la conjoncture, l'organisation qui leur servait à faire la politique.

Les mesures appliquées par le régime autoritaire à partir de 1976 ont constitué un coup brutal à l'exercice des pratiques antérieures, dans la mesure où elles cherchaient la destruction de la base même du pouvoir syndical. C'est ainsi que le nouveau régime entraîna une profonde modification de la législation des associations professionnelles, mît sous tutelle la Confédération générale du travail (C.G.T.), quelques 200 syndicats ainsi que les œuvres sociales, une des sources principales du pouvoir des bureaucraties syndicales. En effet, en novembre 1979 on promulguait la loi 22105 et en août 1980 la loi 22269 dite d'œuvres sociales.

La loi 22105 dissolvait les associations corporatistes des travailleurs du troisième niveau (C.G.T.) et interdisait aux syndicats de participer à des activités politiques, de prêter appui, directement ou indirectement à des partis, des candidats politiques ou à tous ceux qui menaient des activités politiques. L'interdiction pesait également sur l'intervention des syndicats dans la direction de l'administration des œuvres sociales, ainsi que sur la perception de subsides directs ou indirects.

Juridiquement la seule fonction échue aux associations corporatistes des travailleurs était la défense des intérêts économiques 
de leurs membres mais, dans la pratique, la répression militaire, ajoutée à la violence économique, immobilisait tous les canaux de l'action ouvrière. En effet, avec la suspension des commissions paritaires et la fixation par décret des salaires, les syndicats perdaient même la responsabilité de négocier les revendications tout-à-fait professionnelles de leurs membres.

Cette redéfinition de la place et du rôle des syndicats dans la société réussit à contrôler même ce que les organisations ouvrières faisaient le mieux: la mobilisation de leurs bases pour l'obtention des augmentations des salaires, mobilisations qui, étant dirigées généralement contre l'État, étaient des moyens importants de politisation.

La seule alternative à cette redéfinition du syndicalisme par en haut aurait été de gagner des concessions directement des employeurs. Une telle stratégie aurait eu besoin, cependant, d'une organisation très différente et d'un déplacement du centre du pouvoir syndical. En effet, la négociation directe avec l'employeur aurait demandé l'organisation par entreprise et une structure au niveau local, un déplacement du pouvoir syndical du haut de la pyramide vers les dirigeants locaux, donc d'un nouveau syndicalisme que la gloire du passé a empêché ses acteurs d'imaginer.

\section{L'intervention économique.}

Les politiques économiques de facture néo-libérale appliquées pendant la période autoritaire ont été à la source des transformations importantes dans le marché et la structure de la main-d'œuvre qui se sont répercutées au niveau des bases d'appui des syndicats. La crise de l'industrie sous les effets de la récession, de l'inflation et de l'ouverture du marché national à l'importation de biens manufacturés étrangers, produit une baisse drastique de l'emploi industriel, une augmentation de la main-d'œuvre salariée reliée au secteur des services, et une augmentation des travailleurs à 
leur propre compte, cette dernière situation étant très souvent une alternative au chômage.

La tertiarisation de la main-d'œuvre n'est ni un phénomène original de la société argentine, ni une caractéristique exclusive de la période qui nous intéresse. L'originalité de l'Argentine consiste à suivre les mêmes tendances observées dans les sociétés qui ont atteint une maturité industrielle confortable, malgré la fragilité et l'immaturité de sa propre industrie. Dans le cas qui nous occupe la diminution des salariés dans le secteur industriel est due à un processus de désindustrialisation et non pas à l'augmentation de la productivité industrielle comme conséquence de l'introduction des nouvelles technologies. En 1985 on enregistre, par rapport aux chiffres de $1974^{2}$ une chute de 13,9\% dans le nombre d'établissements industriels, de $21 \%$ dans le P.B.I. industriel et de $12,6 \%$ dans la main-d'œuvre employée dans l'industrie. Le nombre de personnes dans ce secteur passait de 1555538 en 1974 à 1359489 en $1985^{3}$ pour une population économiquement active d'environ 10 millions de personnes. En 1974 le secteur manufacturier absorbait $15,51 \%$ de la population économiquement active occupée, tandis qu'en 1985 cette proportion n'était que de $11,85 \%$, une perte d'environ $4 \%$ en onze ans ${ }^{4}$. Le commerce et les services absorbaient les pertes d'emploi de l'industrie et créaient des nouveaux postes pour permettre l'incorporation d'une partie des nouveaux travailleurs suite à la croissance démographique dans la période. Cependant entre 1970 et 1980 le secteur des salariés dans son ensemble enregistrait une perte de $1 \%$ de ses effectifs, la proportion des salariés dans la population économiquement active passait de

2. Censo Econoómico, 1985. Instituto Nacional de Estadística y Censos. República Argentina.

3. Idem.

4. Clarín económico, Buenos Aires, dimanche le 12 janvier, 1986. 
$72 \%$ (chiffre qui s'était maintenu assez stable depuis 1947) à $71 \% 5$.

Depuis les années 50 on constate en Argentine une augmentation importante des travailleurs à leur compte. Entre 1947 et 1980 , cette catégorie de travailleurs passait de $10 \%$ à presque $24 \%{ }^{6}$ de la population économiquement active. Cette tendance s'amplifie particulièrement à partir de 1976; en effet entre cette date et 1980 les travailleurs à leur compte augmentèrent à un taux moyen annuel de 45 pour mille tandis que la population active totale diminuait à un taux de -7 pour mille annuel ${ }^{7}$. En 1975 les travailleurs à leur compte dans le Grand Buenos Aires représentaient $15 \%$ de la population active totale tandis qu'en 1980 ils représentaient $19,7 \%$ de cette population ${ }^{8}$. Mais tandis qu'avant 1976 ce phénomène s'expliquait par les possibilités d'obtenir des revenus plus intéressants en tant que travailleur indépendant plutôt qu'en tant que salarié, après 1976 il s'explique comme une alternative au chômage.

Le taux de chômage est resté assez faible pendant toute la période; dans la capitale fédérale et le Grand Buenos Aires il n'a pas dépassé $5 \%$ et encore entre 1979 et 1980, il se situait autour de $3 \%$. Le chômage a été beaucoup plus élevé dans les autres provinces, mais il ne s'agit pas d'une caractéristique originale de la période autoritaire, les variations pourcentuelles ont suivi le même rythme qu'on observe dans la région où l'activité écoomique se trouve concentrée. Une des explication de ce maintien des taux traditionnels de chômage est sûrement la redistribution de la

5. Ces pourcentages ont été calculés à partir des données fournies par Anuario Estadístico de América latina 1984, CEPAL, 1985.

6. Idem note 4 .

7. Coyuntura y Desarrollo, n. 34, FIDE, juin 1981.

8. Anuario Estadístico de América latina 1983, CEPAL, 1984.

9. Economic Survey of Latin America and the Caribbean: 1982, vol. 1, CEPAL, Santiago, 1984. 
répartition de la population économiquement active selon la situation dans la profession; à cette explication on pourrait ajouter une autre à caractère démographique. En effet, à partir de 1976 le gouvernement impose des restrictions à l'immigration en provenance des pays voisins tout en procédant à l'expulsion, en usant de contraintes juridiques ou économiques, des immigrants frontaliers. Ce phénomène a sûrement contribué à minimiser le chômage interne.

Les politiques de stabilisation du régime autoritaire ne se sont cependant pas limitées à l'ouverture de l'économie aux marchés mondiaux à travers la diminution des tarifs douaniers et la modification des règlements sur les flux du capital étranger. Un autre élément très important de ces politiques a été la distribution régressive des revenus dont les conséquences ont été particulièrement importantes en ce qui concerne le salaire industriel. La chute du salaire réel pourrait constituer un élément complémentaire, quoi que difficile à mesurer, pour expliquer les changements de la structure de la main-d'œuvre; les bas salaires auraient pu favoriser la mobilité des travailleurs entre les différentes catégories d'emploi, notamment de la catégorie des salariés à celle des travailleurs à leur compte.

Après une année d'application du plan de stabilisation par le régime autoritaire, le salaire réel chuta de plus de $30 \%$ et, malgré une légère récupération pendant les années 1979 et 1980, il resta pendant toute la période très au-dessous des valeurs connues en 1975 .

Maintenant nous pouvons nous interroger sur les relations possibles entre les interventions politique et économique du régime auprès des syndicats et leur perte relative du pouvoir dans la société. Étant donné l'importance des transformations économiques qui se sont produites dans la période, la tentation d'expliquer les comportements des acteurs à partir de changements structurels 
TABLEAU 1

Évolution du salaire réel $(1975-1982)^{10}$

\begin{tabular}{lcc}
\hline & $\begin{array}{c}\text { Pouvoir adquisitif du salaire réel } \\
\text { Base 1975 }=100 \\
\text { (d'après Carta Económica) }\end{array}$ & $\begin{array}{c}\text { Salaires moyens réels } \\
\text { Base 1975 = 100 } \\
\text { (d'après CEPAL) }\end{array}$ \\
\hline 1975 & 100,0 & 100,0 \\
1976 & 68,2 & 67,3 \\
1977 & 54,3 & 66,3 \\
1978 & 56,7 & 65,1 \\
1979 & 61,7 & 74,8 \\
1980 & 72,2 & 83,6 \\
1981 & 65,4 & 74,7 \\
1982 & 55,7 & 67,0 \\
\hline
\end{tabular}

est grande. Cependant l'analyse des relations entre le niveau structurel et celui des comportements implique nécessairement un autre niveau d'analyse. Il s'agit des transformations de la propre structure des syndicats et de la Centrale syndicale pendant la période. En effet, dans ce travail il est question de l'action ouvrière à travers les organisations des travailleurs: il faut donc s'interroger à propos des conséquences des transformations du marché du travail sur la composition de ces organisations. En d'autres termes, est-ce que les changements structurels que nous avons relevé pendant la période autoritaire auraient eu un impact semblable dans la composition de la Centrale syndicale qui pourrait expliquer l'affaiblissement du pouvoir syndical?

Depuis les années 50 le secteur industriel dépassa en nombre le secteur tertiaire dans le mouvement ouvrier organisé, ce qui reflétait la distribution réelle de la force de travail dans les différents secteurs d'activité. La supériorité de la représentation des travailleurs industriels au sein de la Confédération Générale du Travail a permis le déplacement de l'hégémonie sectorielle; les syndicats 1986.

10. Julio Sevares, "Los salarios en la Argentina», El Bimestre, n. 26, CISEA, 
d'industrie déplacent les syndicats de services dans le contrôle de la centrale ouvrière et deviennent la force dynamique du syndicalisme argentin. Les données sur la représentativité sectorielle des syndicats ne semblent pas relever des changements radicaux de tendance entre les années 1976 et 1986. Elles démontrent, cependant, que les transformations au niveau de la situation dans la profession de la main-d'œuvre se manifestent déjà aussi bien dans la structure numérique que dans l'ordre des principaux syndicats. En effet, parmi les dix premiers syndicats d'après le nombre d'affiliés, sept se retrouvent aussi bien en 1976 qu'en 1986; aux deux dates, on retrouve à peu près le même nombre de syndicats d'industrie que de syndicats de services, de commerce et de finances, ces derniers étant toujours très importants parmi les dix plus grands. Ces données ne font que confirmer la représentativité sectoriale traditionnelle des syndicats depuis les années 50 . L'importance de la représentation des syndicats non-industriels parmi les dix premiers n'a cependant pas empêché les syndicats représentant les travailleurs de la manufacture de contrôler la Centrale ouvrière. Les raisons qui expliquent ce phénomène sont multiples, mais pour les besoins de cette étude nous n'en avancerons que deux: les syndicats de l'industrie étaient d'une part majoritaires dans l'ensemble du mouvement ouvrier organisé et, d'autre part, les conditions historiques de leur développement ont été à la base d'une formidable unité idéologique. Ces deux caractéristiques des syndicats industriels les placent dans une situation privilégiée quand il était question de la direction de la Centrale ouvrière.

Malgré le fait que la représentation par secteur d'activité parmi les dix premiers syndicats reste assez constante, quatre syndicats industriels (en incluant les cheminots) et six syndicats non-industriels en 1986, contre cinq syndicats de chaque secteur en 1976, le nombre des travailleurs appartenant aux syndicats d'industrie a chuté sensiblement. En effet, en 1976, 52\% des 
TABLEAU 2

Les dix premiers syndicats d'après le nombre d'affiliés et le secteur d'activité économique

\begin{tabular}{|c|c|c|c|c|c|}
\hline \multicolumn{3}{|c|}{1986} & \multicolumn{3}{|c|}{1976} \\
\hline Syndicat & N. Aff. & Secteur & Syndicat & N. Aff. & Secteur \\
\hline $\begin{array}{l}\text { Employés de } \\
\text { commerce }\end{array}$ & 450.000 & commerce & $\begin{array}{l}\text { Personnel civil } \\
\text { de la nation }\end{array}$ & 198.278 & services \\
\hline $\begin{array}{l}\text { Ouvriers } \\
\text { métallurgie }\end{array}$ & 280.000 & industrie & Construction & 175.000 & industrie \\
\hline $\begin{array}{l}\text { Travailleurs } \\
\text { de l'éduc. }\end{array}$ & 250.000 & services & $\begin{array}{l}\text { Employés de } \\
\text { commerce }\end{array}$ & 171.000 & commerce \\
\hline Alimentation & 148.783 & industrie & Cheminots & 170.604 & transport \\
\hline Cheminots & 143.304 & transport & Ouv. du textile & 137.250 & industrie \\
\hline Bancaires & 137.707 & finances & Ouv. métallurgie & 125.000 & industrie \\
\hline $\begin{array}{l}\text { Pers. civil } \\
\text { de la nation }\end{array}$ & 133.188 & services & Trav. de l'État & 124.285 & services \\
\hline Construction & 128.507 & industrie & Bancaires & 76.601 & finances \\
\hline $\begin{array}{l}\text { Travailleurs } \\
\text { État }\end{array}$ & 86.000 & services & Mécaniciens auto. & 75.850 & industrie \\
\hline $\begin{array}{l}\text { Employés de } \\
\text { restaurants }\end{array}$ & 85.481 & services & $\begin{array}{l}\text { Ouv. et employés } \\
\text { municipaux (UOEM) }\end{array}$ & 65.000 & services \\
\hline
\end{tabular}

Source: les données sur le nombre d'affiliés ont été tirées des actes des élections syndicales déposées au Ministère du travail de la République Argentine. Les données de 1986 correspondent aux élections qui ont eu lieu entre 1985 et 1986; les données de 1976 correspondent aux élections qui ont eu lieu entre 1972 et 1976 selon le syndicat. Pour les deux périodes il s'agit de chiffres fournis par les syndicats.

travailleurs des dix premiers syndicats étaient des ouvriers industriels, tandis qu'en 1986 ils ne représentaient que 35\% de la même population. Parmi les syndicats non-industriels, l'augmentation la plus importante du nombre d'affiliés, plus de $150 \%$, se retrouve chez les employés de commerce (Federación de empleados de comercio); un autre phénomène significatif est l'émergence d'un syndicat de travailleurs de l'éducation unitaire (Confederación de trabajadores de la educación) qui réunit des nouveaux et des anciens syndicats autrefois dispersés. Parmi les syndicats industriels 
les seuls à avoir augmenté significativement leur nombre d'affiliés sont ceux de la métallurgie (Unión obrera metalúrgica) qui fut le syndicat le plus puissant depuis 1955 et celui de l'alimentation (Federación de trabajadores de la industria de la alimentación). Deux des syndicats dont la participation a fortement marqué la période 1955-1976, celui des ouvriers du textile (Associación obrera textil) et celui des ouvriers de l'industrie automotrice (Sindicato de mecánicos y afines del transporte automotor) ne font plus partie en 1986 de la liste des dix premiers syndicats.

Cette analyse démontre qu'il existe une association assez étroite entre les transformations de la structure occupationnelle produites par les politiques économiques du gouvernement autoritaire et la représentativité sectorielle parmi les dix premiers syndicats d'après le nombre d'affiliés. Cette constatation permet d'affirmer que l'affaiblissement du pouvoir syndical qui s'est manifesté à partir de la libéralisation et de l'ouverture du régime des militaires a été déterminé, au moins en partie, par le bouleversement de la structure productive et par la rupture radicale avec les politiques économiques du passé. Cependant elle ne permet pas de départager avec exactitude l'efficacité de chacune des interventions, politique et économique.

Au moins objectivement le gouvernement avait réussi sa stratégie face à la classe ouvrière organisée: affaiblir les bases d'appui du pouvoir syndical. Est-ce que la pratique syndicale populiste, qui s'était montrée tellement efficace, surtout dans les années 60 , n'était plus qu'un souvenir du passé?

\section{Action syndicale, pouvoir syndicale et ouverture politique}

Force est de constater que les syndicats n'ont pas relevé le défi que leur tendait la nouvelle conjoncture. Les changements structurels et la politique répressive du régime autoritaire se sont 
traduits par l'affaiblissement du pouvoir syndical, non accompagné, cependant, des stratégies de rechange par rapport à l'action syndicale traditionnelle.

La réponse à l'attaque du régime fut une longue léthargie qui ne fut interrompue que vers 1979 par la réaction des syndicats les plus touchés par la récession et par des discussions aux sommets sur la tactique à suivre face au gouvernement: critiquer ou essayer de collaborer. Ceci ressemblait à d'autres situations connues depuis 1955, où le mouvement ouvrier se trouvait divisé, concernant les attitudes à prendre envers des gouvernements militaires; d'après la tactique préconisée il y avait eu des «durs», des «mous», des «collaborationnistes», des "participationnistes», etc...

La mobilisation populaire qui avait abouti à la troisième présidence de Perón, avait été à la base du surgissement de nouvelles tendances qui avaient disputées le pouvoir aux bureaucraties syndicales au nom d'une gestion différente des affaires internes et d'un nouveau type d'action syndicale. Le nettoyage des «indésirables», les directions de ces nouvelles tendances, avait été commencé avec la bénédiction du vieux leader et continué par les militaires. La libéralisation du régime permet donc l'émergence du vieux syndicalisme, avec ses bases malmenées par la crise et la répression, mais toujours prêt à reprendre la stratégie qui avait été employée avec succès depuis 1955.

Après cinq ans de repli l'année 1982 fut marquée par une reprise de la contestation ouvrière. Cette reprise suivit l'affaiblissement du gouvernement autoritaire et elle s'accentua à partir de la guerre des Malouines qui avait permis aux dirigeants ouvriers de récupérer leur visibilité. En effet, la réapparition de l'action syndicale s'inscrit dans le contexte de crise politique du régime. Les dirigeants syndicaux retrouvèrent des scénarios qui leur étaient 
plus familiers: un gouvernement traversé par des conflits apparemment insolubles qui laissaient la place au dialogue et aux pressions revendicatives.

Juste avant la guerre des Malouines, en février 1982, la CGT représentante de la ligne «dure», publia un programme d'action et se déclara en «état d'urgence»; quelques jours plus tard elle appela à une manifestation pour le 30 mars. Cette manifestation eut lieu trois jours avant le déclenchement des opérations aux Malouines et, malgré la faible participation des bases (entre 30000 et 50000 personnes selon les sources), elle fut violemment réprimée.

La guerre des Malouines mît entre parenthèses les revendications proprement corporatistes. Au même titre que l'ensemble de la société civile, les organisations ouvrières furent amenées à se prononcer sur la légitimité de l'action entreprise par les militaires ce qui donna à la CGT des arguments extra-économiques pour mobiliser ses bases. L'appel à manifester l'appui à la reconquête de la souveraineté politique sur les îles australes eut plus d'écho que l'appel du 30 mars.

Le 7 avril le ministre du Travail invita le Secrétaire général de la CGT, S. Ubaldini, à discuter la situation créée par le conflit avec l'Angleterre et à organiser des voyages de dirigeants syndicaux à l'extérieur avec l'objet de publiciser la position argentine. En même temps, la CGT et les 62 organisations ${ }^{11}$ appellèrent à une mobilisation des travailleurs pour défendre la souveraineté et en appui aux forces armées ${ }^{12}$. Cependant, les revendications des manifestants débordaient largement les raisons qui les avaient amenés à la Place de Mai; la mobilisation fut plutôt une occasion de manifester contre le gouvernement.

11. Regroupement des syndicats péronistes à l'intérieur de la CGT.

12. El Bimestre, N 3, CISEA, 1982. 
La CGT et la CNT-20 ${ }^{13}$ firent preuve d'opportunisme et profitèrent de l'ouverture que leur tendait le gouvernement, malgré lui. En effet, malgré les critiques entendues à la Place de Mai, le gouvernement avait besoin de la mobilisation populaire pour montrer à l'Angleterre et à ses alliés (plus nombreux que les militaires l'auraient pensé) que les Malouines étaient une revendication qui faisait l'unanimité en Argentine. Les militaires avaient gouverné surtout en démobilisant, donc quoi de mieux pour garantir la mobilisation que les organisations ouvrières. Ces dernières conscientes de l'importance qu'elles gagnaient subitement aux yeux des militaires, et de la possibilité inédite de manifester qui leur donnait la conjoncture, commencèrent à radicaliser rapidement leurs demandes. Les changements qui suivirent la défaite des Malouines donnèrent aux dirigeants syndicaux le cadre politique nécessaire pour pratiquer le dialogue.

Le premier juillet 1982 le général Reynaldo Bignone, dernier président du «Proceso», prît la direction d'un gouvernement qui, après des échecs politique, économique et militaire, était complètement affaibli. Le contrôle de l'activité syndicale fut relâché et, dans la pratique les deux CGT, Brasil et Azopardo, formée par la CNT et d'autres groupes, furent reconnues malgré la loi des Associations syndicales promue par les militaires. En août 1982 le gouvernement annulait par décret la loi qui avait enlevé les œuvres sociales aux syndicats et Héctor Villaveiran, ministre de travail, admettait que la majorité de la législation syndicale se devait d'être modifiée ${ }^{14}$.

Le nouveau ministre du travail se montra vite disposé à faire des concessions aux dirigeants syndicaux; les normalisations syndicales s'accéléraient, pendant que le gouvernement octroyait des

13. Groupement syndical qui répondait aux secteurs le plus dialoguistes. Les conflits entre les différents groupes a amené, dans la pratique, à la constitution de deux CGT.

14. Latin America, Regional Report, Southern Cone, le 4 février 1983. 
augmentations de salaires non prévues dans ses énoncés de politiques. La nouvelle légalité n'avait pas seulement des conséquences positives pour l'establishment syndical, elle réouvrait aussi de vieilles rivalités et en provoqua de nouvelles. Aux conflits entre les deux CGT s'ajoutait celui avec les non-alignés et les tensions issues de la restructuration des 62 organisations péronistes. En plus, la majorité des leaders syndicaux des deux centrales syndicales étaient impliqués dans la réorganisation interne du parti péroniste qui ne s'annonçait pas facile. Les luttes pour le pouvoir l'emportaient une fois de plus sur la lutte pour la défense de la condition de vie et de travail des bases représentées.

En mai 1983 celui qui était à l'époque le pré-candidat présidentiel de l' «Unión Civica Radical» (Union civique radicale, UCR), Raúl Alfonsin, dénonçait l'existence d'une entente secrète entre les dirigeants syndicaux et les forces armées. D'après cette entente, le gouvernement laisserait les grands syndicats aux dirigeants durs en échange de leur indulgence face aux abus de pouvoir, notamment face à la répression. Malgré la condamnation de ces affirmations de la part des centrales syndicales, des 62 organisations et des dirigeants politiques du péronisme, il n'est pas exclu que le rapprochement que nous avons signalé entre les syndicalistes et le gouvernement, surtout le ministre du travail, ait eu des dérivations politiques plus larges. Il ne s'agirait pas de la première fois où le syndicalisme cherchait une alliance avec les militaires, ou que ces derniers faisaient appel à l'appui syndical. Les alliances civico-militaires comme instrument pour obtenir le pouvoir politique n'ont pas été en Argentine le seul monopole des groupes de la bourgeoisie. Au contraire, cette forme d'accès au gouvernement a été très souvent envisagée par les organisations ouvrières et cela malgré les maigres résultats obtenus chaque fois qu'une telle alliance s'est minimalement concrétisée. En paraphrasant 
A. Rouquié ${ }^{15}$, en Argentine les militaires nouent des relations avec les partis (voir les syndicats) pour se faire reconnaître et les gouvernements successifs tentent d'obtenir des armées une légitimité qui semble souvent décisive. Il y aurait donc, une militarisation de la vie publique et une politisation sui-generis des forces armées.

Quel était l'enjeu de cette nouvelle alliance civico-militaire?

Pour les syndicalistes, l'enjeu était double; il s'agissait d'avoir les faveurs du gouvernement dans une lutte électorale qui s'annonçait difficile aussi bien au niveau des élections internes du parti péroniste qu'au niveau des élections nationales. Au premier niveau, en tirant des concessions aux militaires en échange d'une attitude indulgente envers leur gestion, ils pouvaient démontrer aux forces non-syndicales du péronisme, qu'ils étaient les seuls capables d'attirer les votes nécessaires pour gagner les élections. Cela veut dire qu'ils pouvaient s'assurer d'une position privilégiée aussi bien dans les postes de direction du parti que dans les listes de candidats aux élections nationales. Il s'agissait, donc, d'imposer l'hégémonie syndicale à l'intérieur du péronisme. Au niveau national, leur bonne conduite pouvait leur garantir une loi électorale qui les favoriseraient.

Quant aux militaires, ils s'étaient joué, sans succès, le tout pour le tout dans la guerre des Malouines; leur départ étant imminent, il s'agissait d'essayer d'imposer des conditions à leur retraite, notamment celles qui leur garantiraient qu'ils ne seraient pas jugés pour les crimes perpétrés pendant leur gestion.

Le scénario ressemblait à celui d'autres campagnes électorales, la stratégie du syndicalisme restait constante; les dirigeants essayaient d'obtenir de l'État une légitimité qui leur semblait décisive.

15. Rouquié, Alain: L'État militaire en Amérique latine, Paris, Seuil, 1982. 


\section{La défaite politique du syndicalisme}

La victoire électorale du Parti radical en octobre 1983 marque le premier échec important de la stratégie utilisée par les syndicalistes depuis 1955. En effet, sans proscription aucune, le parti péroniste perd les élections ${ }^{16}$.

La défaite électorale fut inattendue pour les dirigeants syndicaux; les vieilles tactiques corporatistes se montrèrent inefficaces et le gagnant fut un parti qui n'avait derrière lui aucune puissante corporation et qui n'avait pas eu recours à l'appui de l'État pour se légitimer ${ }^{17}$.

Les causes de l'échec électoral du péronisme ne se réduisent pas seulement au comportement des syndicats. Il est vrai que le syndicalisme n'a pas su ou n'a pas pu adapter sa stratégie à une société profondément convulsionnée par sept ans de gouvernement autoritaire. En outre le prolétariat industriel, son appui principal, s'était réduit en nombre et en force, et la réorganisation sociale produite par le «Proceso» avait eu un effet important sur les formes de participation politique antérieures à 1976.

Les changements dans les formes de participation politique se sont exprimés, en Argentine, par une partidisation de la politique, par l'émergence d'une nouvelle scène publique et par une nouvelle

16. Depuis 1955 les gouvernements, civils ou militaires, s'étaient servis de la proscription électorale pour empêcher le péronisme de prendre le pouvoir. Entre 1955 et 1982 trois gouvernements furent élus démocratiquement. En 1958 Arturo Frondizi, candidat de la Unión Cívica Radical Intransigente, est élu président de la Nation avec le péronisme proscrit mais avec son appui électoral. En 1963 Arturo Illia, candidat de la Unión Cívica Radical del Pueblo, est élu avec l'aide de la proscription du péronisme; il n'obtient que $25,25 \%$ des votes exprimés. À l'époque le mot d'ordre du péronisme proscrit avait était de voter en blanc; les votes en blanc ont représenté $19,4 \%$ des votes exprimés. En 1973 le péronisme remportait les élections présidentielles malgré la proscription qui pesait sur la candidature de Juán Perón.

17. Raúl Alfonsín à la tête de la Unión Cívica Radical a obtenu 7659530 votes, soit plus du $50 \%$ du total exprimé. Le candidat péroniste a obtenu presque 6 millions de voix et le Partido Intransigente qui est arrivé en troisième lieu, 344434 votes. 
relation avec la politique. Comme le signale García Delgado ${ }^{18}$, le processus de transition en Argentine s'est caractérisé par «la prédominance de la participation en partis par rapport à d'autres espaces», par «l'émergence de nouvelles formes de participation» aussi bien au niveau national qu'au niveau local, et par «la modification des attitudes envers le système politique».

La crise du régime autoritaire produit la mobilisation des larges secteurs de la population, notamment des classes moyennes non identifiées soit avec les corporations, soit avec d'autres acteurs dont les formes de participation se situaient à l'extérieur du système partisan. En effet, la participation corporatiste avait exclu toute participation autonome des secteurs non-corporatistes de la vie politique, à moins de subordonner leurs intérêts à ceux des premiers. Une des principales caractéristiques de la période de transition est que l'accent fut mis sur la politique, sur la recherche d'un consensus, ce qui a atténué, au moins en partie, les revendications catégorielles, corporatistes et même de classe. Cette dominance du politique a eu comme résultat une certaine égalisation des demandes qui se structuraient, principalement, autour de la démocratie et des droits humains, les deux demandes à faire le plus fort consensus et qui impliquaient l'existence du pluralisme, d'une éthique politique et de l'État de droit. Malgré leur fragilité, les partis étaient mieux armés que les corporations pour répondre à ce type de demandes. Ils pouvaient se présenter comme les représentants des intérêts collectifs de la Nation, comme les défenseurs des valeurs bafouées et dont la récupération était l'affaire de tous et non pas seulement de quelques groupes en particulier.

18. Daniel R. Garcia Delgado, «Nuevos patrones de participación política en procesos de transición a la democracia: el caso argentino", in Oszlak, Oscar y otros, "Proceso" crisis y transición democrátical2, Buenos Aires, Centro Editor de América Latina, 1984, p. 88-132. 
La crédibilité du discours éthique et démocratique, pour l'électorat en général, a varié en fonction du passé et de la composition interne de chaque parti. Dans cette perspective, les avantages du radicalisme d'Alfonsín furent de deux ordres. D'une part, il se situait en rupture avec son propre parti, avec le radicalisme historique, qui depuis les années 40 s'était défini plus par son antipéronisme que par un projet politique de rechange. D'autre part, le manque d'appui corporatiste qui avait été son principal handicap, surtout entre les années 1955 et 1976, devenait un atout dans la conjoncture de la transition. En effet, le discours radical ne s'attaquait pas seulement au corporatisme militaire, mais il dénonçait aussi celui des syndicats et prônait la construction d'une société pluraliste où les intérêts des groupes non corporatistes auraient une place dans le système politique. Le radicalisme promettait la destruction de ce corporatisme à l'envers qui caractérisait la société Argentine, où ce n'était pas l'État qui organisait les groupes d'intérêts, mais plutôt ces derniers qui l'organisaient ou le désorganisaient. Il s'agissait d'une revendication de la démocratie et du système de représentation à travers les partis politiques que les syndicats interprétaient, et non sans raisons, comme des atteintes à leur propre pouvoir politique.

Le Parti péroniste, au contraire, n'a pas pu surmonter son identification corporatiste ainsi que son image autoritaire malgré les contenus démocratiques et éthiques du discours de son candidat à la présidence, Italo Luder. Parallèlement à ce discours, celui des syndicalistes était tout autre, l'incitation verbale à la violence ainsi que la mise en application des pratiques plus qu'autoritaires dans la réorganisation interne du parti.

Avec plus ou moins de crédibilité, les partis politiques sont devenus les dépositaires de nouvelles revendications qui s'étaient 
exprimées, avant la transition, par des nouvelles formes de participation: organisations de femmes ${ }^{19}$, mouvements de défense des droits humains, mouvement de rock national. Il s'établit une large solidarité fondée sur des valeurs qui privilégiaient le droit à la vie comme condition de l'ordre politique ${ }^{20}$. Les partis n'ont pas pu faire abstraction de cette nouvelle scène publique ce qui les a amenés à introduire leurs revendications en tant qu'aspects centraux de leurs plateformes électorales.

Pendant que les valeurs éthiques et les revendications politiques monopolisaient la scène de la transition, des transformations importantes émergeaient dans la manière de faire la politique. En effet, la transition entre le gouvernement militaire de la période 1966-1973 et le troisième gouvernement péroniste, avait été marquée par l'opposition armée au régime incarné par des différents groupes de guérilla. Par contre, l'opposition contre le dernier régime autoritaire se fit au nom de la légalité, du respect de la Constitution, contre la violence, l'autoritarisme et l'exclusion sociale.

La période de la transition a donc été marquée par le développement des éléments démocratiques d'une culture politique dont les aspects autoritaires furent plutôt dominants. Les syndicalistes, de par leur passé et de par l'inertie actuelle d'une tradition associée à des pratiques plus ou moins autoritaires, perdaient dans la lutte politique une partie de leurs alliés naturels.

19. Les femmes, «en contestant les prétensions du régime autoritaire de redéfinir les limites de l'action politique, se sont organisées»... «à partir de la reconnaissance du profil politique des rôles familiaux". Le groupe le plus visible de cette nouvelle "scène publique» fut, sans conteste, celui des Mères de la Place de Mai qui même symboliquement ont occuppé la place de la politique en manifestant face au palais du gouvernement. (Voir, à ce sujet, María del Carmen Feijoó et Mónica Gogna. "Las mujeres en la transición a la democracia", in Jelin, Elizabeth (comp.), Los nuevos movimientos sociales/1. Buenos Aires, Centro editor de América Latina, 1985. note 18 .

20. Cette problématique est traitée en profondeur par García Delgado, op. cit.. 


\section{Conclusion}

Des ruptures et des constantes ont marqué l'ouverture politique du régime autoritaire en Argentine ainsi que la période de transition qui a mené à la récupération des institutions démocratiques. En ce qui concerne la classe ouvrière, les ruptures se sont exprimées aussi bien au niveau de l'affaiblissement du pouvoir syndical que de la perte de pouvoir de convocation à l'intérieur et à l'extérieur du parti auquel elle était massivement identifiée. L'ouverture fut le produit de la décomposition du régime où la classe ouvrière organisée, pour la première fois depuis 1955, n'a pas été le protagoniste principal du changement. La transition la trouva dépourvue face aux ruptures, provoquées en grande partie par le régime, aussi bien au niveau de ses propres bases qu'au niveau de la société dans son ensemble.

Les politiques du régime autoritaire avaient réussi à contrôler les organisations ouvrières à travers l'intervention politique, ainsi qu'à affaiblir les bases d'appui du pouvoir syndical au moyen de mesures économiques qui ont produit des transformations importantes dans le marché et dans la structure de la main d'œuvre. La baisse significative de l'emploi industriel s'est réflétée dans les syndicats d'industrie qui depuis les années 50 constituaient la force hégémonique à l'intérieur du syndicalisme argentin.

Malgré les changements structurels et politiques introduits par sept ans de gouvernement autoritaire, les syndicalistes n'ont pas proposé des alternatives à l'action traditionnelle. D'une part, ils ont eu des énormes difficultées à surmonter la logique corporatiste, d'autre part ils ont essayé d'obtenir, de l'État, en l'occurrence les militaires, la légitimité qui leur semblait décisive.

Cependant la défaite électorale du syndicalisme, via le parti péroniste, n'est pas uniquement l'expression des changements objectifs chez la classe ouvrière et des constantes observées dans le type d'action qu'il a privilégié. 
La réorganisation sociale issue du régime autoritaire a produit des changements dans les formes de participation politique, changements qui se sont manifestés, notamment, par une partidisation de la politique et par une certaine uniformisation des demandes. Le consensus se fît spontanément autour de la démocratie et des droits humains. Malgré leur fragilité, les partis pouvaient mieux répondre à ce type de demandes que les corporations, d'autant plus que ces dernières étaient tenues responsables, à des degrés divers, de la reproduction de l'autoritarisme.

La transition fut la période de la pure politique et de la politique pure. La pure politique laissait de côté les revendications catégorielles et/ou classistes et plaçait en deuxième lieu ce qui aurait pu atténuer les solidarités. La politique pure a eu la capacité d'imposer et ses valeurs et son discours, ainsi que de provoquer des solidarités actives dans une société démobilisée et repliée sur elle-même comme conséquence de la crise et de la terreur.

À partir d'une perspective régionale quelques conclusions, importantes pour le futur de ces démocraties, s'imposent. En premier lieu, la transition en Argentine fut le produit d'une rupture et non pas d'une réforme comme dans les cas du Brésil et de l'Uruguay. Cette rupture produite par la débâcle du régime n'a pas eu comme contrepartie une opposition forte au niveau de la société civile. En deuxième lieu et comme conséquence du type de transition, les changements dans les formes de faire la politique furent les produits des comportements spontanés des électeurs, indépendants des décisions ou de pactes entre les élites civiles entre elles ou entre les élites civiles et les militaires. Le rôle de l'électorat n'a pas été seulement important au moment même du vote, mais aussi dans l'élaboration des contenus programmatiques des partis, les anciens partis ${ }^{21}$ ont récupéré ou formulé le discours

21. Voir Bruneau Th. et Faucher Ph. Back to Politics: The Forced Path to Regime Change in Latin America and Southern Europe. GRAL, Université de Montréal, 1987. 
majoritaire de l'ensemble de la société. Aussi bien au Brésil qu'en Uruguay le rôle de l'électorat a pu être plus ou moins important seulement au moment de l'élection même. En troisième lieu l'espace politique en Argentine, pendant la transition, a été récupéré par des structures qu'y avaient été toute-à-fait secondaires, les partis politiques. Les corporations, au moins transitoirement, se voyaient retranchées derrière leur fonction économique. Au Brésil et en Uruguay, par contre, il n'y a pas de bouleversement, les élites civiles et les partis politiques reprennent les fonctions qui avaient été les leurs avant l'autoritarisme.

Tout ceci a fait que la situation post-électorale ait été plus incertaine en Argentine qu'au Brésil et en Uruguay. Il n'y a pas eu de pacte implicite comme au Brésil ou explicite comme l'Accord du Club Naval en Uruguay, entre les facteurs de pouvoir. Ils n'ont pas souscrit des accords qui auraient engagé la responsabilité des acteurs dans leur application. En d'autres termes, aucun des facteurs de pouvoir se compromettait apriori, à respecter des normes de fonctionnement de la société qui permettraient la consolidation de la démocratie. Ces normes de fonctionnement ont commencé à se définir à posteriori, c'est-à-dire une fois les radicaux au pouvoir. La question militaire et la concertation sociale accaparent depuis 1984 le débat politique en Argentine, cependant, les rapports actuels entre ceux qui auraient du être les acteurs de la concertation permettent de penser que les anciennes traditions d'affrontement l'emportent sur les politiques de construction d'un consensus. La conjoncture démocratique constitue une fois de plus le terrain propice pour l'application des tactiques de pression sociale de la part des corporations. La récomposition de l'ordre politique semble plus ardue dans la mesure où les pactes sociaux ne sont pas un préalable aux élections mais, au contraire, un processus entamé par après, présidé par un État politisé dont la bannière partisane n'est pas celle des corporations, elles aussi fortement politisées. 